\title{
HEMOGLOBINA Y TESTOSTERONA: IMPORTANCIA EN LA ACLIMATACIÓN Y ADAPTACIÓN A LA ALTURA
}

\author{
Gustavo F. Gonzales ${ }^{1, a}$
}

\begin{abstract}
RESUMEN
Los diferentes tipos de mecanismos que emplea el organismo cuando se enfrenta a una situación de hipoxia incluyen la acomodación, la aclimatación y la adaptación. La acomodación es la respuesta inicial a la exposición aguda a la hipoxia de altura y se caracteriza por aumento de la ventilación y de la frecuencia cardiaca. La aclimatación se presenta en los individuos que están temporalmente expuestos a la altura y, que en cierto grado, les permite tolerar la altura. En esta fase hay un incremento en la eritropoyesis, se incrementa la concentración de hemoglobina y mejora la capacidad de transporte de oxígeno. La adaptación es el proceso de aclimatación natural donde entra en juego las variaciones genéticas y la aclimatación que les permiten a los individuos vivir sin dificultad en la altura. La testosterona es una hormona que regula la eritropoyesis y la ventilación, podría estar asociada con los procesos de aclimatación y adaptación a la altura. La eritrocitosis excesiva que conduce al mal de montaña crónico es causada por una baja saturación arterial de oxígeno, una ineficiencia ventilatoria y reducida respuesta ventilatoria a la hipoxia. La testosterona se incrementa en la exposición aguda en la altura y en los nativos de altura con eritrocitosis excesiva. Los resultados de las investigaciones actuales permitirían concluir que el incremento de la testosterona y de la hemoglobina son buenas para la aclimatación adquirida pues mejoran el transporte de oxígeno pero no para la adaptación a la altura, dado que valores altos de testosterona en suero se asocian con eritrocitosis excesiva.
\end{abstract}

Palabras clave: Testosterona, Eritropoyesis, Altitud, Aclimatación, Adaptación Fisiológica (fuente: DeCS BIREME).

\section{HEMOGLOBIN AND TESTOSTERONE: IMPORTANCE ON HIGH ALTITUDE ACCLIMATIZATION AND ADAPTATION}

\begin{abstract}
The different types of response mechanisms that the organism uses when exposed to hypoxia include accommodation, acclimatization and adaptation. Accommodation is the initial response to acute exposure to high altitude hypoxia and is characterized by an increase in ventilation and heart rate. Acclimatization is observed in individuals temporarily exposed to high altitude, and to some extent, it enables them to tolerate the high altitudes. In this phase, erythropoiesis is increased, resulting in higher hemoglobin and hematocrit levels to improve oxygen delivery capacity. Adaptation is the process of natural acclimatization where genetical variations and acclimatization play a role in allowing subjects to live without any difficulties at high altitudes. Testosterone is a hormone that regulates erythropoiesis and ventilation and could be associated to the processes of acclimatization and adaptation to high altitude. Excessive erythrocytosis, which leads to chronic mountain sickness, is caused by low arterial oxygen saturation, ventilatory inefficiency and reduced ventilatory response to hypoxia. Testosterone increases during acute exposure to high altitude and also in natives at high altitude with excessive erythrocytosis. Results of current research allow us to conclude that increase in serum testosterone and hemoglobin is adequate for acclimatization, as they improve oxygen transport, but not for high altitude adaptation, since high serum testosterone levels are associated to excessive erythrocytosis.
\end{abstract}

Key words: Testosterone, Erythropoiesis, Altitude, Acclimatization, Physiological Adaptation (source: MeSH NLM).

\section{INTRODUCCIÓN}

En el mundo, más de 140 millones de personas habitan permanentemente en zonas por encima de los 2500 metros de altura (1) en condiciones de hipoxia debido a la baja presión parcial de oxígeno, que determina importantes cambios en el organismo en la que destaca el incremento en la producción de glóbulos rojos ${ }^{(2)}$.
Esta exposición permanente a la altura contrasta con la observada por aquella población que residiendo a nivel del mar debe ascender a diferentes alturas ya sea por trabajo, deporte o turismo.

Ambas exposiciones a la baja presión barométrica - la del nativo de altura y la exposición aguda a la altura representan situaciones diferentes con enfermedades

\footnotetext{
Jefe de la Unidad de Reproducción, Instituto de Investigaciones de la Altura y Jefe del Laboratorio de Endocrinología y Reproducción, Facultad de Ciencias y Filosofía, Universidad Peruana Cayetano Heredia.

a Doctor en Medicina y Doctor en Ciencias. Especialista en Endocrinología.
}

$$
\text { Recibido: 21-12-10 Aprobado: 02-02-11 }
$$


diferentes aunque con muy grandes homologías, entre ellas, el incremento en la producción de los glóbulos rojos. Entre las diferencias podemos anotar que el sujeto expuesto agudamente a la altura se caracteriza por un aumento inmediato en la ventilación ${ }^{(3)}$ en tanto que la característica del nativo de altura es la hipoventilación, lo cual conduce a hipoxemia, eritrocitosis excesiva y finalmente en mal de montaña crónico ${ }^{(4)}$. En América muchas poblaciones de ciudades y capitales importantes se ubican en zonas de altitud como en el caso de Leadville $(3045 \mathrm{~m})$ y Denver $(1584 \mathrm{~m})$ en Colorado, Estados Unidos; México DF (2240 m) y Toluca (2680 m) en México; San José de Costa Rica (1160 m); Bogotá (2640 m) en Colombia; Quito (2850 m) en Ecuador; La Paz (3600 m) y Sucre (2904 m) en Bolivia.

En Perú, el $30 \%$ de la población, unos nueve millones de personas, reside en altitudes por encima de 2000 metros. Muchos departamentos cuyas capitales se asientan en zonas de altura superan el millón de habitantes como Junín con su capital Huancayo (3280 $\mathrm{m})$; Cusco con su capital Cusco (3400 m); Puno con su capital Puno (3800 m); Arequipa con su capital Arequipa (2335 m); Cajamarca (2750 m); Ancash con su capital Huaraz (3000 m).

Los diferentes tipos de mecanismos que emplea el organismo cuando se enfrenta a una situación de hipoxia incluyen la acomodación, la aclimatación y la adaptación. La acomodación es la respuesta inicial a la exposición aguda a la hipoxia de altura y se caracteriza por aumento de la ventilación y de la frecuencia cardiaca. La aclimatación se presenta en los individuos que están temporalmente expuestos a la altura y, que en cierto grado, les permite tolerar la altura. En esta fase hay un incremento en la eritropoyesis, se incrementa la concentración de hemoglobina y mejora la capacidad de transporte de oxígeno, se le conoce también como aclimatación adquirida. La adaptación es el proceso de aclimatación natural donde entran en juego las variaciones genéticas y la aclimatación que les permiten a los individuos nacer, crecer y reproducirse en la altura en forma natural y normal. Para conseguir la adaptación se requiere el paso de muchas generaciones ${ }^{(5)}$.

La eritrocitosis excesiva que es causada por una baja saturación arterial de oxígeno, una ineficiencia ventilatoria y reducida respuesta ventilatoria a la hipoxia, es el signo cardinal del mal de montaña crónico; en tanto que la hipertrofia ventricular derecha, la hipertensión pulmonar y el remodelamiento de las arteriolas pulmonares contribuyen a la hipertensión pulmonar inducido por la altura. En la patogénesis de estas afecciones parecen confluir factores genéticos que determinarían que algunas personas que nacen y viven en la altura desarrollen estas enfermedades en tanto que otras no ${ }^{(6)}$.

Desde la descripción inicial de Viault en 1890 (7) de un incremento en el número de glóbulos rojos en los nativos de los Andes Centrales del Perú, se ha planteado la hipótesis si la mayor eritropoyesis es un mecanismo adaptativo a la altura. Cien años después, datos de diferentes autores han sido analizados y verificados por León-Velarde en $1990{ }^{(8)}$ quien en su artículo Evolución de las ideas sobre la policitemia como mecanismo adaptativo a la altura confronta esta hipótesis y ,más bien, propone que la eritrocitosis normal o excesiva es una desventaja para la adaptación a la altura.

Monge y Whittembury (9) desarrollan un modelo matemático donde demuestran que el hombre en la altura no necesita de un alto hematocrito para el transporte de oxígeno máximo, y que por el contrario la eritrocitosis debe considerarse como una adaptación limitada a moderadas altitudes ${ }^{(2)}$, pero una mala adaptación a mayores alturas. Winslow y Monge ${ }^{(10)}$ concluyen que la eritrocitosis excesiva en los nativos de la altura no tiene ninguna utilidad.

Teniendo en cuenta que tanto en la exposición aguda a la altura como en el nativo de altura se observan niveles elevados de hemoglobina/hematocrito y que cuando los valores son muy altos, constituyen una enfermedad denominada eritrocitosis excesiva, que conduce al mal de montaña crónico, es importante conocer cómo se regula la aparición de esta eritrocitosis excesiva. En esta búsqueda se ha estudiado a la eritropoyetina, hormona producida en el riñón, que actúa favoreciendo la eritropoyesis. Efectivamente, en la altura, los niveles de eritropoyetina se encuentran incrementados; sin embargo, en los casos de eritrocitosis excesiva los niveles de eritropoyetina no son diferentes a los de aquellos individuos nativos de altura que no tienen este exceso ${ }^{(11,12)}$. Existe otra hormona, la testosterona ${ }^{(12,13)}$ que actúa sobre la eritropoyesis y cuya elevación en la pubertad determina las diferencias en los niveles de hemoglobina entre hombre y mujeres y cuya función regulatoria de la eritropoyesis en la altura no ha sido aún completamente analizado.

La presente revisión trata de enfocar la función de la testosterona, una hormona con importante efecto eritropoyético ${ }^{(13)}$ en los procesos de aclimatación y adaptación a la altura.

HORMONAS Y ADAPTACIÓN A LA ALTURA

El Texto de Endocrinología de 1985, un clásico para la especialidad, establecía que la respiración no tiene un 
elemento significativo de control endocrino ${ }^{(14)}$. En los siguientes 15 años se acumuló tal cantidad de evidencia sobre la interacción de la respiración y las hormonas, con lo cual dicha aseveración fue eliminada en la edición del mismo texto en $1998{ }^{(15)}$. Hoy en día se acepta que el control de la respiración tiene mecanismos de control voluntario (corteza) e involuntario (componentes emocionales, metabólicos, neural y endocrino) ${ }^{(16)}$.

Las hormonas cumplen una función importante en los procesos de adaptación a cambios ambientales, por lo que no es raro pensar que también cumplan una función en el proceso de adaptación a la altura. Los datos relacionados al mal de montaña crónico (MMC), una enfermedad de falta de adaptación a vivir en la altura, revelan su menor frecuencia en mujeres durante edades premenopáusicas ${ }^{(17)}$, pero aumenta luego de la menopausia (18). Esto indicaría que las hormonas sexuales tendrían un papel en la adaptación a la altura en forma diferencial entre hombres y mujeres.

Una característica del mal de montaña crónico (MMC) es la eritrocitosis excesiva evaluada por un nivel alto del hematocrito o de la hemoglobina ${ }^{(19)}$, que para la zona de Cerro de Pasco $(4340 \mathrm{~m})$ se ha definido como valores de hemoglobina $\geq 21 \mathrm{~g} / \mathrm{dL}$ o de hematocrito $>63 \%$ en varones ${ }^{(20)} y \geq 19 \mathrm{~g} / \mathrm{dL}$ en mujeres ${ }^{(21)}$.

Se reconoce que la principal causa del MMC es la hipoventilación que genera una disminución en la saturación arterial de oxígeno. Esta se asocia con una elevación de los niveles de hemoglobina como mecanismo de compensación de la menor presión parcial de oxígeno y tratando de compensar la llegada de oxigeno a los tejidos. Esta mayor eritrocitosis puede llegar a niveles muy altos que afecta el flujo de sangre a los tejidos produciendo los síntomas y signos observados en el MMC ${ }^{(4,21)}$.

En las Figura 1 se observan datos obtenidos en nuestro laboratorio donde se aprecia el nivel de asociación entre la saturación arterial de oxígeno y el hematocrito en varones adultos de Lima (150 m) y Cerro de Pasco (4340 m).

La mejor correlación entre saturación arterial de oxigeno y hematocrito es polinomial donde la saturación arterial de oxígeno está en valores altos en el grupo con bajos valores de hematocrito, luego, ante una caída de la saturación arterial de oxígeno hay una elevación importante del hematocrito.

La eritropoyesis es un proceso regulado hormonalmente. Al menos dos hormonas tienen las propiedades de inducir la producción de eritrocitos, la eritropoyetina (Epo) ${ }^{(22)}$ y la testosterona ${ }^{(13,23)}$.

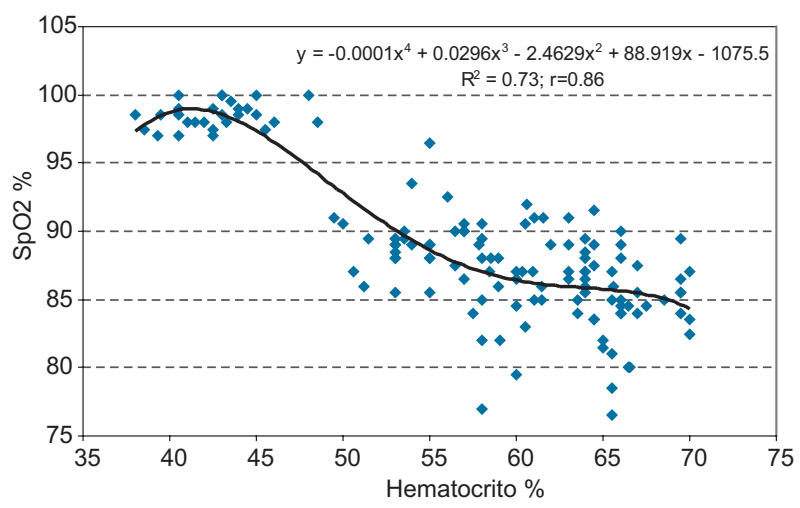

Figura 1. Asociación de saturación arterial de oxígeno por pulsometría con el hematocrito en varones adultos de Lima y Cerro de Pasco (Gonzales y col, no publicado).

La eritropoyetina es la hormona principal que regula la eritropoyesis. La eritropoyetina es una glicoproteína de 30,4 KDa producida principalmente por el riñón ${ }^{(22)}$. La eritropoyetina estimula la proliferación y diferenciación de los precursores eritroides en la médula ósea ${ }^{(24)}$.

En los nativos de los Andes hay una gran variabilidad en los niveles de Epo y la respuesta eritrocítica a la altura; sin embargo, no hay correlación entre los niveles de Epo séricos en nativos de la altura con eritrocitosis excesiva y aquellos viviendo en la altura sin esta condición patológica ${ }^{(11,12)}$.

El hipotiroidismo es otro cuadro clínico asociado con hipoventilación (25). Sin embargo, no se observan diferencias en los niveles de tiroxina o tri-iodotironina en los nativos de Cerro de Pasco, con o sin eritrocitosis excesiva ${ }^{(12)}$.

Si la eritropoyetina no cumple una función principal en el MMC, es posible que la otra hormona eritropoyética, la testosterona tenga un papel preponderante. La testosterona probablemente actúe directamente en la médula ósea a nivel de los eritroblastos policromatofílicos y mejore la síntesis de RNA ribosomal o sus precursores y estimule una ribonucleasa nuclear (23). La administración de testosterona estimula la producción de glóbulos rojos en varones en forma dependiente de la dosis especialmente en la tercera edad (26), y es responsable del aumento de la hemoglobina que ocurre en varones con la pubertad (27).

La testosterona no solo tiene un efecto estimulador de la eritropoyesis sino también inhibe la ventilación (28), mientras que el estradiol inhibe la eritropoyesis y estimula la ventilación ${ }^{(29,30)}$. En ese sentido, un incremento en los niveles de estrógenos circulantes se asocia con mayores valores de saturación arterial de oxígeno ${ }^{\left({ }^{31}\right)}$. A pesar de 
estos hallazgos, no está aún claramente demostrado de manera directa en humanos, que el estradiol inhiba la eritropoyesis, por lo que se requieren de nuevos estudios para clarificar el papel de esta hormona.

La exposición de varones adultos mayores a altas dosis de testosterona se asocia con un menor tiempo total de sueño, mayores episodios hipóxicos y aumento en el índice de trastornos respiratorios (el número de apneas e hipópneas por hora) ${ }^{(32)}$. Estos resultados indican efectivamente que una de las acciones de los andrógenos es producir hipoventilación. Esto no es discordante con el efecto eritropoyético de la testosterona, pues, en resumen la acción de la testosterona sobre la eritropoyesis sería doble: 1) a través de hipoventilación que conduce a reducción en la saturación arterial de oxígeno, hipoxemia y estímulo a la eritropoyesis y 2) efecto directo de la testosterona sobre la eritropoyesis.

\section{TESTOSTERONA Y ERITROPOYESIS}

La testosterona regula la eritropoyesis en diversas especies de mamíferos incluyendo a los humanos de ambos sexos ${ }^{(13)}$. La eritrocitosis excesiva es el principal problema asociado con la administración de testosterona en varones adultos mayores ${ }^{(33)}$. La testosterona parece actuar directa e indirectamente para estimular la eritropoyesis ${ }^{(13,23)}$, también incrementa los niveles de hemoglobina y hematocrito de manera dosis dependiente sin un aumento asociado en los niveles de eritropoyetina (26). Si bien se ha demostrado un efecto de testosterona sobre las células progenitoras eritroides, recientes estudios demuestran que la testosterona también tiene la capacidad de regular la disponibilidad de hierro en el organismo ${ }^{(34)}$. El hierro es un importante componente de la hemoglobina, y su deficiencia conduce a anemia.

La hepcidina, un polipéptido producido en el hígado, es la principal hormona reguladora del hierro que media la homeostasis de las concentraciones extracelulares de hierro ${ }^{(35)}$. La hepcidina actúa regulando la entrada de hierro al plasma a partir de los tejidos: enterocitos duodenales que absorben hierro de la dieta, hepatocitos que almacenan hierro y de los macrófagos que reciclan hierro de los eritrocitos seniles ${ }^{(36)}$. La hepcidina se liga y degrada el transportador en el canal de hierro, ferroportina (37). La elevación de hepcidina resulta en menor absorción intestinal de hierro y con ella menor disponibilidad de hierro para la eritropoyesis. Recientemente se ha demostrado que la testosterona en humanos inhibe los niveles de hepcidina, incrementando con ello la absorción intestinal de hierro ${ }^{(34)}$.

La eritrocitosis es el efecto adverso más frecuente de la administración de testosterona por lo que se supone que la supresión de hepcidina por la testosterona podría cumplir una función importante en esta enfermedad. La mayoría de varones que responden a la testosterona aumentando los niveles de hematocrito y hemoglobina y estables niveles de eritropoyetina, estarían asociados con una disminución en los niveles de hepcidina ${ }^{(34)}$.

\section{ACLIMATACIÓN A LA ALTURA}

Durante la exposición aguda a la altura hay un incremento inmediato de la ventilación y de la frecuencia cardiaca tratando de compensar la menor presión parcial de oxígeno; el aumento de la ventilación se acompaña con una pérdida de $\mathrm{CO}_{2}$ que, a su vez, se traduce en una alcalosis respiratoria.

La ventilación tiene regulación periférica y central. Las principales estructuras envueltas en el control neural de la ventilación incluyen los quimiorreceptores del cuerpo carotídeo, el núcleo del tracto solitario, la columna respiratoria ventral, el núcleo hipogloso en el tallo cerebral, el núcleo motor frénico en la médula espinal y las entradas neuromoduladoras a estas regiones ${ }^{(38)}$. La serotonina cumple una función importante en la regulación de la ventilación ${ }^{(39,40)}$. Estudios en nuestro laboratorio han demostrado un incremento en los niveles de serotonina durante la exposición aguda a la altura. A nivel central se ha asociado una interrelación entre serotonina y testosterona en la regulación de la ventilación ${ }^{(39)}$. Una evidencia adicional de la relación entre la testosterona y la función respiratoria es la presencia de receptores de andrógenos en el pulmón (41). A nivel periférico, la testosterona inhibe la función de los cuerpos carotídeos ${ }^{(42)}$.

La exposición a la altura aumenta los niveles de testosterona sérica y de serotonina sanguínea ${ }^{(43,44)}$. Al parecer la elevación de la testosterona puede ser explicada, en parte, por una regulación serotoninérgica debido a que el uso de un antagonista de la serotonina como la ciproheptadina previene el incremento de la testosterona por la exposición aguda a la altura ${ }^{(43)}$. Es posible que esta elevación de la testosterona contribuya a regular la hiperventilación que se produce por la exposición aguda en la altura. Dado que la testosterona es una hormona hipoventiladora, su elevación durante la exposición aguda a la altura permitiría ejercer un efecto de retroalimentación evitando que la hiperventilación sea excesiva y la alcalosis respiratoria que ella produce genere los síntomas de mal de montaña agudo o soroche. Esto se comprueba cuando se estudian varones expuestos agudamente a la altura de Cerro de Pasco (4340 m). Aquellos que con mayor saturación arterial de oxígeno tienen mayores niveles de testosterona que aquellos con bajos valores de saturación arterial de 


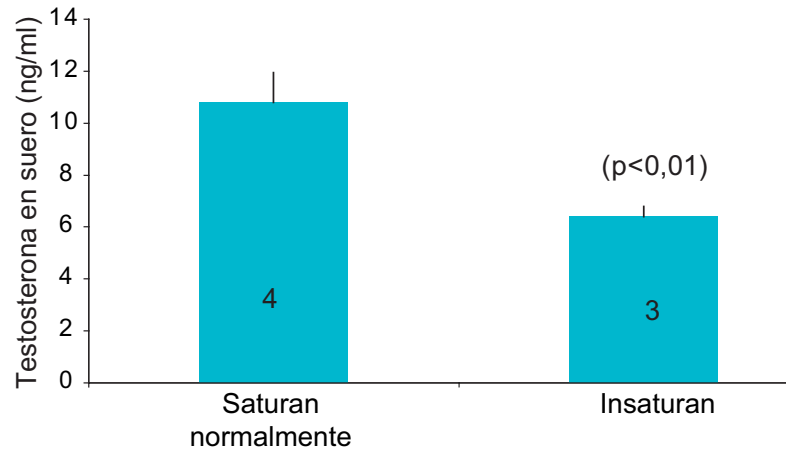

Figura 2. Niveles de testosterona sérica en varones expuestos durante 24 horas a la altura de Cerro de Pasco (4340 m). (Fuente: Gonzales GF, no publicado) ${ }^{*} \dagger$.

* La columna de la izquierda representa a varones con mayores valores de saturación arterial de oxígeno que los de la columna de la derecha $(\mathrm{P}<0,01)$. Los datos son medias \pm error estándar

† Los números dentro de las barras indican el número de sujetos.

oxígeno (Figura 2). La mayor saturación es evidencia de mayor ventilación en el primer grupo de sujetos.

El aumento en los niveles de testosterona sérica durante la exposición aguda a la altura también favorecería el aumento de la eritropoyesis evidenciado en mayor hematocrito ${ }^{(44)}$. En resumen, durante la exposición aguda a la altura hay una respuesta inicial de hiperventilación que busca compensar la menor presión parcial de oxígeno. Con la hiperventilación ocurre una mayor eliminación de $\mathrm{CO}_{2}$ conducente a una alcalosis respiratoria, que al ocurrir, genera sintomatología propia del mal de montaña agudo. Se postula que como mecanismo de regulación la hiperventilación favorece la elevación de testosterona. El incremento de testosterona a su vez reduciría la ventilación; esta elevación de la testosterona también estimularía la producción de glóbulos rojos y con ello mejora el transporte de oxígeno facilitando el proceso de aclimatación.

Estos resultados indicarían que la elevación tanto de la testosterona como de la hemoglobina/hematocrito sería buena para aclimatación a la altura.

\section{ADAPTACIÓN A LA ALTURA}

En un estudio comparativo en Bolivia a 3600 m, varones de zonas urbanas presentaron niveles más altos de testosterona y de hemoglobina que los Aymaras de zonas rurales con mayor antigüedad generacional en la zona altitudinal ${ }^{(45)}$. Esto sugiere que valores de testosterona en el rango normal alto puede comprometer el proceso de adaptación a la altura.

Del mismo modo, en varones de Cerro de Pasco, Perú a $4340 \mathrm{~m}$ se ha observado mayor biodisponibilidad de testosterona, lo que sugeriría que esta población

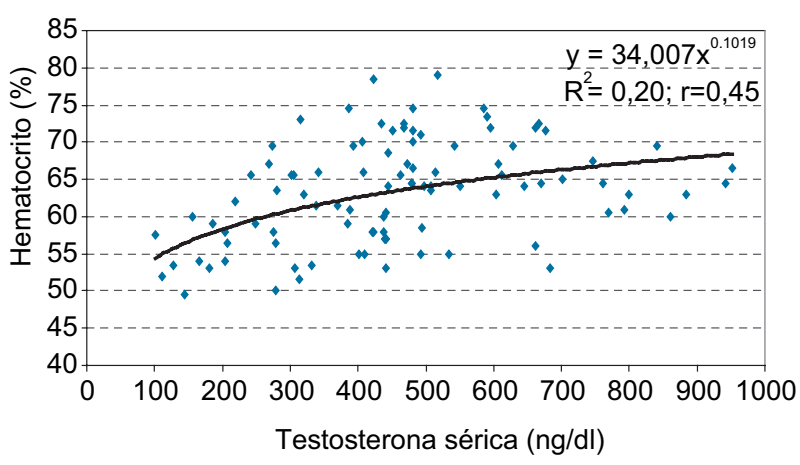

Figura 3. Asociación entre los niveles de testosterona sérica y el hematocrito en varones adultos de Cerro de Pasco (4340 m).

residente en los Andes centrales pueda no haber completado su proceso de adaptación ${ }^{(46)}$. Por lo tanto, la hipótesis que se origina es que la testosterona podría estar relacionada con la eritrocitosis excesiva y, esta a su vez, a la etiopatogenia del mal de montaña crónico. Se sugiere que la eritrocitosis es debida a una mayor concentración plasmática de la relación testosterona/ estradiol, tanto en varones como en mujeres ${ }^{(46-48)}$. La testosterona disminuye la ventilación durante el sueño y favorece la eritropoyesis, en tanto que el estradiol estimula la ventilación e inhibe la eritropoyesis.

Recientemente se ha demostrado que los varones de Cerro de Pasco con eritrocitosis excesiva tienen mayores niveles séricos de testosterona que aquellos viviendo en la misma altitud pero sin eritrocitosis excesiva (12). En la Figura 3 se puede apreciar que efectivamente hay una relación directa entre los niveles de testosterona sérica y el hematocrito. A mayor testosterona hay mayor hematocrito.

En Cerro de Pasco, pero no en Cusco, se ha demostrado una mayor respuesta de testosterona sérica a la hCG (49), tal como se observa en la Figura 4, que indicaría que la zona de Cerro de Pasco es más propensa a la eritrocitosis excesiva que Cusco. La antigüedad generacional es mayor en Cusco que en Cerro de Pasco y ello puede contribuir a que en Cerro de Pasco se presente con mayor frecuencia la eritrocitosis excesiva, o en otras palabras que la población de Cusco se encuentre mejor adaptada que la de Cerro de Pasco a vivir en la altura ${ }^{(50)}$.

Esto también ha sido corroborado en cerca de 6000 residentes en los Himalayas (Tibetanos y Han) donde se demuestra que la mayor eritrocitosis se observa en varones Han y que este efecto se produce después de la pubertad ${ }^{(51)}$. Los Han tienen un poco más de 60 años habitando el Tibet en tanto que los Tibetanos tienen una antigüedad generacional mayor de 25000 años. 


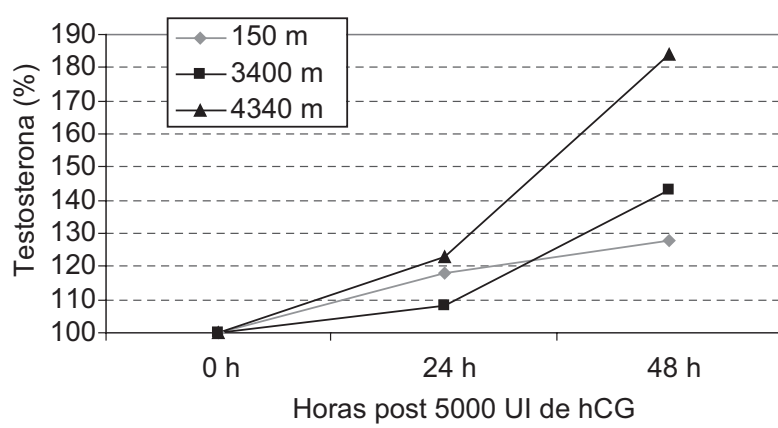

Figura 4. Respuesta de varones de Lima (150 m), Cuzco (3400 m) y Cerro de Pasco (4340 m) al estímulo con $5000 \mathrm{UI}$ de hormona coriónica gonadotropa (hCG) intramuscular ${ }^{(49)}$.

En la Figura 5 se observa que a medida que el puntaje del cuestionario de mal de montaña crónico en varones de Cerro de Pasco (4340 m) es mayor, el valor de testosterona sérica es mayor ${ }^{(52)}$. En este mismo estudio se demuestra que la testosterona elevada en la altura independiente del nivel de hemoglobina se asocia con algunos signos y síntomas de mal de montaña crónico. Ello evidencia la necesidad de no solo corregir los niveles altos de hemoglobina sino más bien los niveles elevados de testosterona con lo que no solo se disminuyen los signos y síntomas dependientes de esta elevación sino aquellos dependientes de la elevación de la hemoglobina.

Más recientemente se ha demostrado que los niveles elevados de testosterona en varones con eritrocitosis excesiva se deberían a un rápido metabolismo de dehidroepiandrosterona sulfato (DHEAS) a androstenediona y de este a testosterona debido a una mayor actividad de la enzima 17 beta hidroxiesteroide deshidrogenasa ${ }^{(12)}$. Esto también parece ocurrir en mujeres, donde los mayores niveles de hematocrito se asocia con niveles elevados de testosterona ${ }^{(48)}$ asociados a valores bajos de DHEAS (Figura 6), que revela la rápida transformación de DHEAS a androstenediona y luego a testosterona.

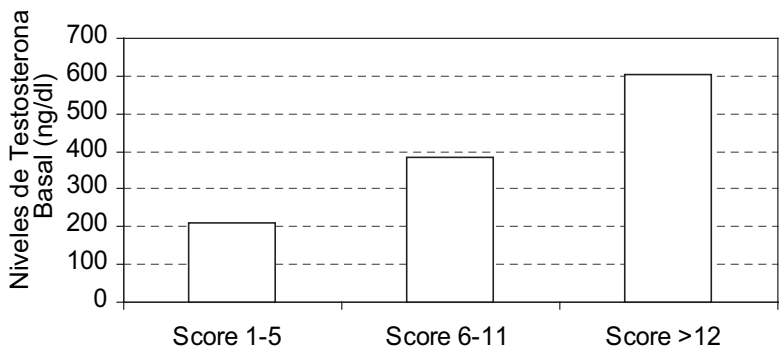

Figura 5. Niveles de testosterona sérica (ng/dL) según puntaje (score) del cuestionario de signos y síntomas de mal de montaña crónico en varones de Cerro de Pasco (4340 m) ${ }^{(52)}$.*

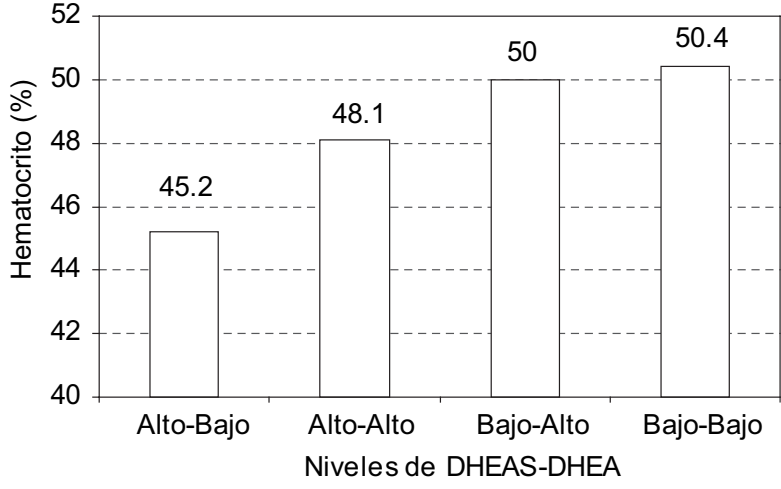

Figura 6. Niveles de hematocrito en mujeres de Cerro de Pasco $(4340 \mathrm{~m})$ según niveles de dehidroepiandrosterona sulfato (DHEAS) y dehidroepiandrosterona (DHEA) (Fuente: Gonzales GF, no publicados)

Los datos analizados sugieren que la eritrocitosis sería un buen mecanismo de aclimatación pero no de adaptación a la altura. El aumento de la hemoglobina en los sujetos de nivel del mar expuestos agudamente a la altura compensaría la menor saturación arterial de oxígeno y facilitaría una aclimatación adquirida. La situación en el nativo de altura es diferente, pues se caracteriza por una menor ventilación y una respuesta atenuada de la ventilación al estímulo hipóxico ${ }^{(4)}$ ello conlleva a estimular la eritropoyesis mediado inicialmente por la eritropoyetina sin que ello implique a llegar a valores de eritrocitosis excesiva $(\mathrm{Hb}>21 \mathrm{~g} / \mathrm{dL}$ en Cerro de Pasco); si el nativo de altura se caracteriza por tener niveles normales altos a valores altos de testosterona en suero, el riesgo de eritrocitosis excesiva es más alta (Figura 7).

Tanto la testosterona como la hemoglobina incrementada se asocian con signos y síntomas de mal de montaña crónico ${ }^{(52)}$. Esto implicaría que la testosterona, siendo buena para aclimatación adquirida a la altura, no lo es para adaptación a la altura (aclimatación natural). De esto se puede decir que la aclimatación adquirida se beneficia del aumento en los glóbulos rojos, en tanto que un individuo adaptado no puede permitirse tener un aumento

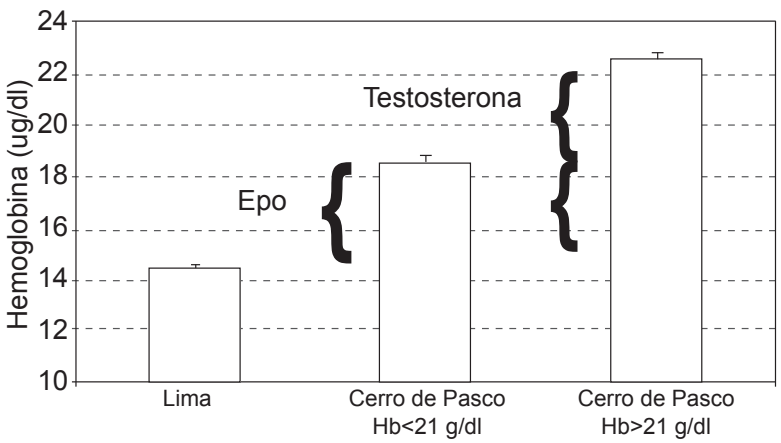

Figura 7. Niveles de hemoglobina en varones de Lima (150 m) y de Cerro de Pasco con $\mathrm{Hb} \leq 21 \mathrm{~g} / \mathrm{dL}$ y con $\mathrm{Hb}>21 \mathrm{~g} / \mathrm{dL}$. (Fuente: Gonzales GF, no publicado)* 
marcado de glóbulos rojos, pues pasado cierto límite produce sintomatología de mal de montaña crónico. De esto se deduce que los varones son más propensos a la eritrocitosis excesiva y al mal de montaña crónico que las mujeres, lo cual ha sido fehacientemente comprobado ${ }^{(4)}$

Los niveles basales de testosterona en suero o plasma han sido estudiados por diversos investigadores. En diversos estudios donde se incluyen varones que no presentan eritrocitosis excesiva, los niveles de testosterona fueron similares a los observados en Lima (150 m). Así, no se han observado diferencias en los niveles de testosterona sérica entre varones de Lima $(150 \mathrm{~m})$ y aquellos sin eritrocitosis excesiva de Cusco $(3400 \mathrm{~m}){ }^{(53,54)}$, Cerro de Pasco (4340 m) ${ }^{(12,55-57)}$ y Morococha $(4540 \mathrm{~m})^{(57)}$.

\section{CONCLUSIONES}

La hiperventilación es un fenómeno que se observa en todos los sujetos que ascienden a la altura (exposición aguda). En el nativo de altura lo que se observa es un fenómeno de hipoventilación. Son dos situaciones diferentes con dos procesos o resultados diferentes. Lo que se postula en la exposición aguda a la altura es que con la hiperventilación hay mayor eliminación de $\mathrm{CO}_{2}$ que conlleva a una alcalosis respiratoria. Si este fenómeno no es regulado la persona tiene síntomas. Se postula que en la exposición aguda a la altura, la hiperventilación genera como respuesta una elevación de testosterona. La testosterona cumple su papel de reducir la ventilación y con ello regular este proceso; además, la testosterona favorece la eritropoyesis y con ello mejora el transporte de oxígeno, lo que conlleva a un proceso de aclimatación.

La situación en el nativo de la altura es otra. Al estar permanentemente estimulado con mayores niveles de testosterona, por ejemplo entre hombres y mujeres, se produce una hipoventilación permanente y este es un estímulo para eritropoyesis que asociado al propio estímulo eritropoyético de la testosterona conduce a la eritrocitosis excesiva y al mal de montaña crónico. Por ello, si bien en situaciones de exposición aguda, la testosterona es útil, no lo es para el nativo de la altura que tiene valores altos de testosterona. Por ello se concluye que la testosterona es buena para aclimatación pero mala para adaptación a la altura.

\section{Fuentes de Financiamiento}

NIH Research Grant \# 5-D43TW005746-04 del Fogarty International Center, National Institutes on Environmental Health Services, National Institute for Occupational
Safety and Health, y Agency for Toxic Substances and Disease Registry.

\section{Conflictos de Interés}

El autor declara no tener conflictos de interés en la publicación del presente artículo.

\section{REFERENCIAS BIBLIOGRÁFICAS}

1. Moore LG. Human genetic adaptation at high altitude. High Alt Med Biol 2001;2: 257-79.

2. Monge CC. Regulación de la concentración de hemoglobina en la policitemia de altura: modelo matemático. Bull Inst Fr Etud Andines (Lima) 1990;19:455-67.

3. Palmer BF. Physiology and pathophysiology with ascent to altitude. Am J Med Sci. 2010;340(1):69-77.

4. Peñaloza $\mathbf{D}$, Arias-Stella J. The heart and pulmonary circulation at high altitudes: healthy highlanders and chronic mountain sickness. Circulation 2007;115:1132-46.

5. Gonzales GF, Villena A. Aclimatación y adaptación. En: El futbol y la aclimatación a la altura. IIAD:Lima. 1998:23-46.

6. León-Velarde F, Mejía $\mathbf{O}$. Gene expression in chronic high altitude diseases. High Alt Med Biol. 2008;9(2):130-9.

7. Viault FG. Physiologie experiméntale: sur augmentation considérable du nombre des globules rouges dans le sang chez les habitants des hauts plateaux de l'Amérique du Sud. CR Séances Acad Sci 1890;111:917-8.

8. León-Velarde F. Evolución de la ideas sobre la policitemia como mecanismo adaptativo a la altura. Bull Inst Fr Etud Andines. 1990; 19:443-53.

9. Monge CC, Whittemburry J. Chronic mountain sickness and the physiopathology of hypoxemic polycythemia. En: Sutton JR, Jones NL, Houston CS (eds), Hypoxia: man at altitude. New York:Thieme Stratton, 1982:51-6.

10. Winslow RM, Monge CC. Hypoxia, polycythemia, and chronic mountain sickness. Baltimore, Md: John Hopkins, 1987: 255 p.

11. León-Velarde F, Monge CC, VidalA, Carcagno M, Criscuolo M, Bozzini CE. Serum immunoreactive erythropoietin in high altitude natives with and without excessive erythrocytosis. Exp Hematol. 1991;19(4):257-60.

12. Gonzales GF, Gasco M, Tapia V, Gonzales-Castañeda C. High serum testosterone levels are associated with excessive erythrocytosis of chronic mountain sickness in men. Am J Physiol Endocrinol Metab. 2009;296:E1319-25.

13. Shahani S, Braga-Basaria $M$, Maggio $M$, Basaria S. Androgens and erythropoiesis: past and present. J Endocrinol Invest 2009,32:704-16.

14. Wilson JD, Foster DW. Introduction. In: Wilson JD, Foster DW, eds. Williams textbook of endocrinology. 7th ed. Philadelphia, PA: W.B. Saunders Company,1985; 1-8

15. Wilson JD, Foster DW, Kronenberg H. Principles of endocrinology. In: Wilson JD, Foster DW, Kronenberg $\mathrm{HM}$, et al, eds. Williams textbook of endocrinology. 9th ed. Philadelphia, PA: W.B. Saunders Company, 1998; 1-10

16. Saaresranta T, Polo $O$. Hormones and breathing. Chest 2002;122:2165-82. 
17. Whittembury J, Monge C. High altitude, hematocrit and age. Nature. 1972;238:278-9.

18. Leon-Velarde F, Ramos MA, Hernandez JA. The role of menopause in the development of chronic mountain sickness. Am J Physiol. 1997;272(1 Pt 2):R90-4.

19. Monge-C C, Leon-Velarde F, Arregui A. Increasing prevalence of excessive erythrocytosis with age among healthy high-altitude miners (Letter). New Engl. J. Med.1989;321:1271.

20. Reeves JT, León-Velarde F. Chronic mountain sickness: recent studies of the relationship between hemoglobin concentration and oxygen transport. High Alt Med Biol. 2004 5:147-55

21. León- Velarde, F, Maggiorini M, Reeves JT, Aldashev A, Asmus I, Bernardi L, Ge RL, Hackett P, Kobayashi T, Moore LG, Peñaloza D, Richalet JP, Roach R, Wu T, Vargas E, Zubieta-Castillo G, Zubieta-Calleja G. Consensus statement on chronic and subacute high altitude diseases. High Alt Med Biol. 2005;6(2):147-57.

22. Richmond TD, Chohan M, Barber DL. Turning cells red: signal transduction mediated by erythropoietin. Trends Cell Biol 2005;15:146-55.

23. Zitsmann M. Effects of testosterone replacement and its pharmacogenetics on physical performance and metabolism. Asian J Androl 2008;10:364-72.

24. Delanghe JR, Bollen M, Beullens M. Testing for recombinant erythropoietin. Am J Hematol 2008;83:237-41.

25. Misiolek M, Marek B, Namyslowski G, Scierski W, Zwirska-Korczala K, Kazmierczak-Zagorska Z, Kajdaniuk D, Misiolek H. Sleep apnea syndrome and snoring in patients with hypothyroidism with relation to overweight. J Physiol Pharmacol 2007;58 Suppl 1:77-85.

26. Coviello AD, Kaplan B, Lakshman KM, Chen T, Singh AB, Bhasin S. Effects of graded doses of testosterone on erythropoiesis in healthy young and older men. J Clin Endocrinol Metab 2008;93:914-9.

27. Gonzales GF, Reynafarje C. Cambios del hematocrito con la pubertad. Diagnóstico. 1982;9:161-78.

28. Favier R, Spielvogel H, Caceres E, Rodriguez A, Sempore B, Pequignot J. Differential effects of ventilatory stimulation by sex hormones and almitrine on hypoxic erythrocytosis. Pflugers Arch 1996;434:97-103.

29. Cristancho E, Reyes O, Serrato M, Mora MM, Rojas JA, Robinson $\mathbf{Y}$, Boning D. Arterial oxygen saturation and hemoglobin mass in postmenopausal untrained and trained altitude residents. High Alt Med Biol 2007;8:296-306.

30. Horiguchi H, Oguma E, Kayama F. The effects of iron deficiency on estradiol-induced suppression of erythropoietin induction in rats: implications of pregnancy-related anemia. Blood 2005;106:67-74.

31. Saaresranta T, Polo-Kantola P, Virtanen I, Vahlberg T, Irjala K, Polo O. Menopausal estrogen therapy predicts better nocturnal oxyhemoglobin saturation. Maturitas 2006;55:255-63.

32. Liu PY, Yee B, Wishart SM, Jimenez M, Jung DG, Grunstein RR, Handelsman DJ. The short-term effects of high-dose testosterone on sleep, breathing, and function in older men. J Clin Endocrinol Metab 2003;88;3605-13.

33. Calof OM, Singh AB, Lee ML, Kenny AM, Urban RJ, Tenover JL, Bhasin S. Adverse events associated with testosterone replacement in older men: a metaanalysis of placebo-controlled trials. J Gerontol Med Sci 2005;11:1451-7.

34. Bachman E, Feng R, Travison T, Li M, Olbina G, Ostland V, Ulloor J, Zhang A, Basaria S, Ganz T, Westerman M, Bhasin S. Testosterone suppresses hepcidin in men: a potentialmechanismfortestosterone-inducederythrocytosis. J Clin Endocrinol Metab. 2010;95(10):4743-7

35. Ganz T, Nemeth E. Iron imports: IV. Hepcidin and regulation of body iron metabolism. Am J Physiol Gastrointest Liver Physiol. 2006;290:G199-203.

36. Ganz T, Olbina G, Girelli D, Nemeth E, Westerman M. Immunoassay for human serum hepcidin. Blood. 2008;112(10):4292-7.

37. Fleming MD. The regulation of hepcidin and its effects on systemic and cellular iron metabolism. Hematology Am Soc Hematol Educ Program 2008:151-8.

38. Feldman JL, McCrimmon DR. Neural Control of Breathing. In: Squire, LR.; Bloom, FE.; McConnell, SK.; Roberts, JL.; Spitzer, NC.; Zigmond, MJ., editors. Fundamental Neuroscience. Vol. 2nd ed. San Diego: Elsevier, Academic Press; 2003:967-990.

39. Behan M, Zabka AG, Thomas CF, Mitchell GS. Sex steroid hormones and the neural control of breathing. Respir Physiol Neurobiol. 2003;136(2-3):249-63.

40. Behan M, Wenninger JM. Sex steroidal hormones and respiratory control. Respir Physiol Neurobiol. 2008;164(1-2):213-21.

41. Mikkonen L, Pihlajamaa $P$, Sahu $B$, Zhang FP, Jänne OA. Androgen receptor and androgen-dependent gene expression in lung. Mol Cell Endocrinol. 2010;317(1-2):14-24.

42. Bairam A, Montandon G, Joseph V, Lajeunesse $Y$, Kinkead R. Enhancement of the breathing frequency response to hypoxia by neonatal caffeine treatment in adult male rats: the role of testosterone. Respir Physiol Neurobiol. 2009;165(2-3):261-5.

43. Gonzales GF, Rodriguez L, Valera J, Sandoval E, and Garcia MA. Prevention of high altitude-induced testicular disturbances by previous treatment with cyproheptadine in male rats. Archives of Andrology. 1990;24:201-5.

44. Gonzales GF, Chung FA, Miranda S, Valdez LB, Zaobornyj T, Boveris A. Heart mitochondrial nitric oxide synthase in rats at high altitude. American Journal of Physiology 2005; 288: H2568-73.

45. Beall CM, Worthman CM, Stallings J, Strohl KP, Brittenham GM, Barragan $\mathbf{M}$. salivary testosterone concentration of Aymara men native to 3600 m. Ann. Hum. Biol. 1992; 19: 67-78.

46. Gonzales GF. Endocrine testicular function and adaptation to high altitude. Newsletter ISMM. 1998; 8: 5-7.

47. Gonzales GF. Hematocrit values in women at high altitude and its relationship with sex hormone levels. Journal of Qinghai Medical College. 2004,25:267-72.

48. Gonzales GF, Villena A. Low pulse oxygen saturation in post-menopausal women at high altitude is related to a high serum testosterone/estradiol ratio. Int J Gynaecol Obstet. 2000 71:147-54.

49. Gonzales GF, Villena A, Llaque W, Kaneku L, Coyotupa J, Guerra-García R. Función endocrina testicular en la 
exposición aguda a la altura y en el nativo andino. Rev Per Endocrin. Metab. 1997;3:62-76.

50. Gonzales GF. Peruvian contributions to the study on human reproduction at high altitude: from the chronicles of the Spanish conquest to the present. Respiratory Physiology \& Neurobiology. 2007; 158:172-9.

51. Wu T, Wang $X$, wei $C$, Cheng $H$, Wang $X$, Li $Y$, Dong G, Zhao H, Young P, Li G, Wang Z. Hemoglobin levels in Qinghai-Tibet: different effects of gender for Tibetans vs Han. J Appl Physiol. 2005;98:598-604.

52. Gonzales GF, Tapia V, Gasco M, Gonzales-Castañeda C. Serum Testosterone levels and score of Chronic Mountain Sickness in Peruvian men natives at $4340 \mathrm{~m}$. Andrologia 2011. en prensa.

53. Garmendia F, Valdivia H, Castillo O, Ugarte N, Garmendia A. Función endocrina en el hombre normal de mediana altitud. I Jorn Medicina y Cirugía de Altura. La Oroya: Perú. Resumen 1:1978.

54. Gonzales GF. Fisiología reproductiva en la altura. En: Reproducción Humana en la Altura. Ed. Gustavo F. Gonzales. Ediciones IIA: Lima, Perú. 1993:3-24.
55. Coyotupa J. Maduración sexual en Cerro de Pasco (4340 m) y Lima (150 m). [Tesis Doctoral] Universidad Peruana Cayetano Heredia. Lima, Perú. 1986.

56. Kaneku L. Respuesta testicular a la hCG en varones jóvenes y ancianos a nivel del mar y en la altura. [Tesis de maestría]. Universidad Peruana Cayetano Heredia: Lima. 1989

57. Llaque W. Estudio del eje hipotálamo-hipofisiario-gonadal en hombres normales a nivel del mar y en la altura. [Tesis Doctoral]. Universidad Peruana Cayetano Heredia. Lima, Perú. 1974

Correspondencia: Gustavo Gonzales Rengifo.

Dirección: Universidad Peruana Cayetano Heredia Facultad de Ciencias y Filosofía, Av. Honorio Delgado 430, San Martín Porras, Lima, Perú.

Teléfono: 999-98072.

Correo electrónico: gustavo.gonzales@upch.pe

\section{Suscríbase en forma electrónica y gratuita a los contenidos de la Revista Peruana de Medicina Experimental y Salud Pública, ingrese a WWW.ins.gob.pe, seleccione el ícono de la revista y envíenos sus datos.}

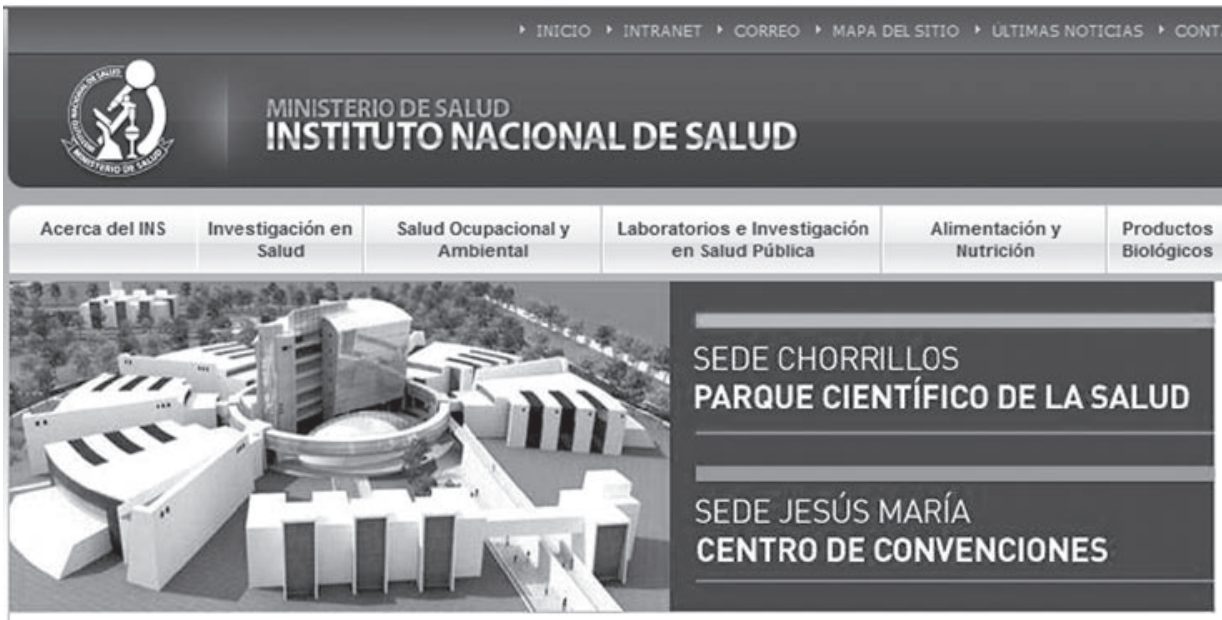

ÚLTIMAS INVESTIGACIONES Y EVIDENCIAS CIENTÍFICAS Ver Todas

Distribución de serotipos del virus dengue Perú 2011 Serotipos de dengue identificados por RT-PCR-TR en el Perú 2011 Ver detalle

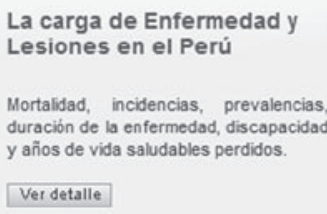

La carga de Enfermedad y Lesiones en el Perú

Mortalidad, incidencias, prevalencias, duración de la enfermedad, discapacida y años de vida saludables perdidos. Ver detalle

GALERÍA

Ver Todas

Imagen del dia 2011

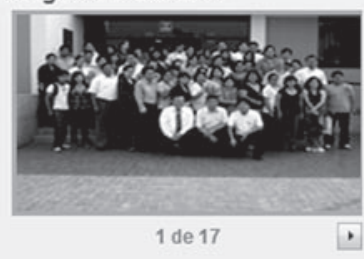

Salud Control de Calidad Intercultural de Medicamentos

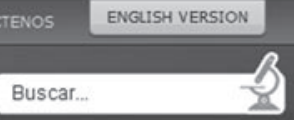

Q Portal del Transparencia

N Gestión de calidad

Directorio Institucional

12. 30 marzo del 2011

Agregar a favoritos

- Enviar a un amigo

Publicaciones

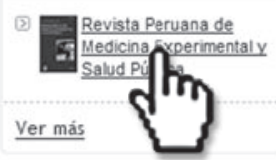

Comités del INS

(2) Comté Especial del Sistema de Control lnterno 